\title{
Computational prediction of muon stopping sites: a novel take on the unperturbed electrostatic potential method
}

Simone Sturniolo and Leandro Liborio

\section{Published version information}

Citation: S Sturniolo and L Liborio. 'Computational prediction of muon stopping sites: a novel take on the unperturbed electrostatic potential method.' Journal of Chemical Physics, vol. 153, no. 4 (2020): 044111.

DOI: $\underline{10.1063 / 5.0012381}$

This article may be downloaded for personal use only. Any other use requires prior permission of the author and AIP Publishing.

This version is made available in accordance with publisher policies. Please cite only the published version using the reference above. This is the citation assigned by the publisher at the time of issuing the APV. Please check the publisher's website for any updates. 


\section{Computational prediction of muon stopping sites: A novel take on the unperturbed electrostatic potential method}

Cite as: J. Chem. Phys. 153, 044111 (2020); https://doi.org/10.1063/5.0012381

Submitted: 01 May 2020 . Accepted: 02 July 2020 . Published Online: 24 July 2020

Simone Sturniolo (iD), and Leandro Liborio (D)
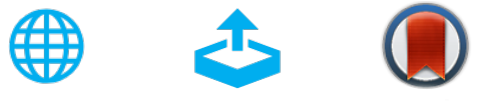

\section{ARTICLES YOU MAY BE INTERESTED IN}

Extended Koopmans' theorem in the adiabatic connection formalism: Applied to doubly hybrid density functionals

The Journal of Chemical Physics 153, 044109 (2020); https://doi.org/10.1063/5.0010743

Combining phonon accuracy with high transferability in Gaussian approximation potential models

The Journal of Chemical Physics 153, 044104 (2020); https://doi.org/10.1063/5.0013826

$\mathrm{N}_{3}^{+}$: Full-dimensional ground state potential energy surface, vibrational energy levels, and dynamics

The Journal of Chemical Physics 153, 044302 (2020); https://doi.org/10.1063/5.0011957
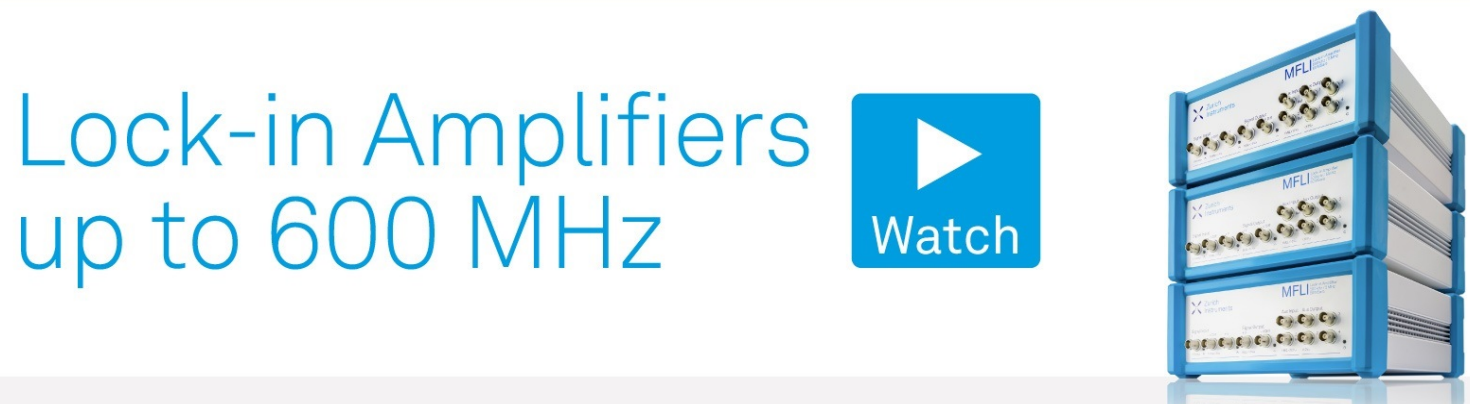


\title{
Computational prediction of muon stopping sites: A novel take on the unperturbed electrostatic potential method
}

\author{
Cite as: J. Chem. Phys. 153, 044111 (2020); doi: 10.1063/5.0012381 \\ Submitted: 1 May 2020 - Accepted: 2 July 2020 • \\ Published Online: 24 July 2020
}

Simone Sturniolo (D) and Leandro Liborio ${ }^{\text {a) }(D)}$

\section{AFFILIATIONS}

Theoretical and Computational Physics Group, Scientific Computing Department, Rutherford Appleton Laboratory, STFC, Harwell Campus, Didcot OX11 OQX, United Kingdom

a) Author to whom correspondence should be addressed: leandro.liborio@stfc.ac.uk

\begin{abstract}
Finding the stopping site of the muon in a muon-spin relaxation experiment is one of the main problems of muon spectroscopy, and computational techniques that make use of quantum chemistry simulations can be of great help when looking for this stopping site. The most thorough approach would require the use of simulations, such as Density Functional Theory (DFT), to test and optimize multiple possible sites, accounting for the effect that the added muon has on its surroundings. However, this can be computationally expensive and sometimes unnecessary. Hence, in this work, we present a software implementation of the Unperturbed Electrostatic Potential (UEP) Method: an approach used for finding the muon stopping site in crystalline materials. The UEP method requires only one DFT calculation, necessary to compute the electronic density. This, in turn, is used to calculate the minima of the crystalline material's electrostatic potential and the estimates of the muon stopping site, relying on the approximation that the muon's presence does not significantly affect its surroundings. One of the main UEP's assumptions is that the muon stopping site will be one of the crystalline material's electrostatic potential minima. In this regard, we also propose some symmetry-based considerations about the properties of this crystalline material's electrostatic potential, in particular, which sites are more likely to be its minima and why the unperturbed approximation may be sufficiently robust for them. We introduce the Python software package pymuon-suite and the various utilities it provides to facilitate these calculations, and finally, we demonstrate the effectiveness of the method with some chosen example systems.
\end{abstract}

Published under license by AIP Publishing. https://doi.org/10.1063/5.0012381

\section{INTRODUCTION}

In a muon-spin spectroscopy experiment, spin-polarized positive muons are implanted in a sample and the pattern of positron emission, caused by the muons' decay, is used to measure the magnetic properties of the host material. Positrons are emitted in the direction of the muon's spin at the time of its decay, and the observation of the decay's time evolution can be used to infer information about the magnetic structure of the sample.

The muon has a half-life of $2.2 \mu \mathrm{s}$, and during this time, it interacts with magnetic moments of either nuclear or electronic origin. These interactions can be used to obtain information on, for instance, local magnetic fields on a small length scale. Muons are used often as a microscopic probe to measure both static and dynamic magnetic ordering. Due to the local nature of these interactions, predicting where the muon would stop inside the host material is one of the key problems of muon-spin spectroscopy, and it has been the subject of many research works. ${ }^{1}$

In our previous work, we presented a set of computational approaches for finding the muon stopping site that could be applied to the case of finding the stopping site of muonium, the pseudo-atom formed by a positive muon when it captures an electron. This set of approaches requires the use of many randomly initialized calculations, based on either the Density Functional Theory (DFT) approximation $^{5}$ or the much faster, lower level approximation of Density Functional Tight Binding (DFTB). ${ }^{6}$ The DFTB approach is by far the faster of the two, but it depends on parameterizations fitted on more fundamental theory calculations that are not available for all elements of the periodic table. ${ }^{7,8}$ Furthermore, both approaches require a certain level of computational power and have a learning curve 
associated with knowing how to properly run a DFT or DFTB simulation. However, for diamagnetic muons-namely, positive muons that have not formed a bound state with an electron and whose energetics are thus dominated by their unshielded electrostatic interaction with the surrounding environment-a much simpler alternative to find the stopping site is available: the unperturbed electrostatic potential method.

The Unperturbed Electrostatic Potential (UEP) method works by assuming that the muon's presence does not significantly affect the crystal order. In this assumption, one can use the known distribution of positive charges (the ions) and the negative electronic charge cloud as found, for example, via a single DFT calculation on the unperturbed system, to compute the Coulomb electrostatic potential felt by the muon at each point in the crystal. It is then possible to compute the forces acting on the muon and which sites constitute a local or absolute minimum of the potential, without running any more expensive calculations.

The general consensus at the time of writing is that the UEP method works better in metals, ${ }^{1,9}$ where the diffuse electronic charge shields the muon effectively at very short ranges, thus preventing it from having a significant effect on the atomic positions. Conversely, if the host material is an insulator or a semiconductor, the stopping sites for the muon can be in an off-center interstitial position because of the formation of chemical bonding between the muon and its neighboring atoms. In this case, the unperturbed electrostatic potential is not a satisfying methodology for finding the muon stopping site any more. This is observed, for example, in lithium fluoride, where the muon forms a $\mathrm{F}-\mu$-F structure by distorting the lattice locally.

In addition to the UEP, we also consider the importance of symmetry considerations for determining the muon stopping sites. In general, it is considered a good rule of thumb in crystallography that sites with high symmetry are often the preferred atomic sites. ${ }^{1}$ This holds true for muons as well, and in practice, very often, the muon stopping site will be a high-symmetry site of the crystal that has not already been occupied by another atom. For this reason, a crystallographic analysis of the pure system that will be studied with muons is a useful first step when looking for muon stopping sites as such analysis is significantly faster than any computer simulation. In this paper, we will outline some specific considerations about the properties we can predict for the electrostatic potential at these high-symmetry sites, and we will also introduce the software implementations of both the symmetry analysis and UEP in the Python library pymuon-suite. Finally, in Sec. IV, we will show the results of both approaches for some choice example systems.

\section{THEORY}

\section{A. Symmetry analysis}

A crystal structure is defined by its symmetry properties. Any crystal, to be called such, must possess at least a translational symmetry since it is fundamentally the infinite repetition of a finite unit cell. However, many crystals possess higher symmetry than that, having a group $\mathscr{G}$ of symmetry operations $(\mathbf{W}, \mathbf{w})$ that connect crystallographically equivalent points,

$$
Y=g(X)=\mathbf{W} X+\mathbf{w}, \quad g \in \mathscr{G} .
$$

In some cases, some points $X$ can be identified for which there exists a subset $S_{X} \subseteq \mathscr{G}$ of operations such that $g(X)=X \forall g \in S_{X}$; these points are called special Wyckoff positions. Wyckoff positions need not be single points; for example, the rotation axis is the Wyckoff position of a rotation operation as it is all left unchanged by it. In this case, one can say that the Wyckoff position has one free coordinate. However, we are here concerned with those Wyckoff positions that have no free coordinates and the symmetry operations under which they are unchanged. In particular, let $X$ be a special Wyckoff position such that $g(X+\varepsilon) \neq X+\varepsilon \forall g \in S_{X}$, with $\varepsilon$ being an arbitrarily small vector, which is a rigorous formulation of the condition that the position has no free coordinates. It then follows that, for an arbitrary function $f(X)$ that has the same symmetry properties as the crystal, such as the electrostatic potential, $X$ has to be a stationary point, namely, $\nabla f(X)=0$.

The proof of this is the following. Consider the transformation properties of the gradient under a symmetry operation. In general, we have that $X_{i}^{\prime}=W_{i j} X j+w_{i}$, and so,

$$
\nabla f=\partial_{i} f=\partial_{j}^{\prime} f \partial_{i} X_{j}^{\prime}=\partial_{j}^{\prime} f W_{j i}=\nabla^{\prime} f \mathbf{W} .
$$

In a special Wyckoff position, however, $\nabla f=\nabla^{\prime} f$. Then, it must be

$$
\nabla f(X)=\nabla f(X) \mathbf{W} \quad \forall(\mathbf{W}, \mathbf{w}) \in S_{X} .
$$

For Eq. (3) to remain valid at the special Wyckoff position $X$, while the value of the gradient at that same position is different from zero, there should be at least one direction that is invariant under the entire group of operations $S_{X}$. However, we already established that this is not the case as the Wyckoff position $\mathrm{X}$ has no free coordinates. Therefore, the solution must be the trivial one, i.e., the value of the gradient of an arbitrary function $f(X)$ at position $X$ is zero.

This already gives us a hint as to why special Wyckoff positions with no free coordinates are good candidates for the muon stopping site: because they are necessarily stationary points of the electrostatic potential. This is not even restricted only to the unperturbed system; it may still hold even if the muon introduces some deformation, provided that said deformation does not break the symmetries contained in $S_{X}$. However, a stationary point of a function is not guaranteed to be a minimum: it could still be a maximum or a saddle point and, in general, is not possible to determine which one is just from symmetry considerations. However, it is possible to perform a stricter series of checks on $S_{X}$ to identify which Wyckoff positions put further constraints not only on the gradient but also on the second derivatives of the function, the so-called Hessian. In fact, with a similar reasoning as the one here presented for the gradient, one can find that in some points, the function is constrained to have a Hessian that is either positive or negative definite (and thus, the function has either a minimum or a maximum), and in even more special cases, the Hessian is isotropic, and the function must have locally radial symmetry. The details of this derivation are included in Appendix A.

\section{B. UEP}

The core assumption of the unperturbed electrostatic potential method is that the muon experiences a potential

$$
V(\mathbf{x})=\int_{\mathbb{R}^{3}}\left[\rho_{e}(\mathbf{r})+\rho_{I}(\mathbf{r})\right] \frac{1}{|\mathbf{r}-\mathbf{x}|} d \mathbf{r},
$$


where the integral is carried out over all the (infinite) volume of the crystal, and the charge density has been split in electronic $\left(\rho_{e}\right)$ and ionic $\left(\rho_{I}\right)$ contributions. It can be seen easily that this integral is hard to converge in real space as the Coulomb potential only falls off as $1 / r$, whereas the Jacobian ${ }^{12}$ in spherical coordinates goes like $r^{2}$. In fact, the integral would not converge at all if the net charge was not guaranteed to be zero. In practice, this is always guaranteed as the system without the muon is neutral and the total charge of each unit cell is zero. However, it is far more practical to compute the integral in Fourier space, where

$$
V(\mathbf{x})=\frac{4 \pi}{v} \int_{\mathbb{R}^{3}}\left[\rho_{e}(\mathbf{G})+\rho_{I}(\mathbf{G})\right] \frac{e^{i \mathbf{G x}}}{|\mathbf{G}|^{2}} d \mathbf{G}
$$

and convergence is faster. Here, $v$ is the volume of a single unit cell, $v=(\mathbf{a} \times \mathbf{b}) \mathbf{c}$ in terms of the lattice parameters.

The current implementation of the UEP method in pymuonsuite is compatible only with CASTEP calculations, though support for other codes will be developed in the future. CASTEP is a DFT code using a plane wave basis set and pseudopotentials. The plane wave basis means it is most suited to treat periodic solids, and here, we can make use of that peculiarity by noting that the electronic density returned by it can be expressed as a truncated Fourier series, so the electronic contribution to the potential $V_{e}$ can be written as

$$
V_{e}(\mathbf{x})=\frac{4 \pi}{v} \sum_{\mathbf{G}>0} \rho_{e}(\mathbf{G}) \frac{e^{i \mathbf{G} \mathbf{x}}}{|\mathbf{G}|^{2}},
$$

where the sum runs over a finite number of reciprocal vectors $\mathbf{G}$ that depends on the cutoff used for the calculation. Ideally, when preparing the calculation, one should have taken care that this value is high enough to properly converge the energy of the system, which means the truncation should not affect the final value much as $\rho_{e}(\mathbf{G})$ will go to zero for large $\mathbf{G}$. Note that we ignore $\mathbf{G}=0$, where Eq. (6) would diverge, because this term represents the total charge, and while we know that independently it will not be zero for both $\rho_{e}$ and $\rho_{I}$, we also know it will eventually cancel out when we sum them as that represents the fact that the system is overall neutral.

The problem remains then of how to deal with $\rho_{I}$. This is the charge density including both the positive charge $Z_{i}$ of each atomic nucleus and the negative one of its $N_{i}$ core electrons that CASTEP embeds in the pseudopotential. We choose here to simplify the problem by assuming that this charge can be treated as a purely Gaussian charge distribution, centered on the atomic position and of width $\sigma_{i}$ that we base off some measure of the expected radius of the ion. Currently, the software uses $\sigma_{i}=r_{p p o t} / s$, where $r_{p p o t}$ is the smallest core radius used in the construction of the pseudopotential and $s$ is a user-defined scaling parameter. This leads to the expression

$$
V_{I}(\mathbf{x})=\frac{4 \pi}{v} \sum_{i}\left(Z_{i}-N_{i}\right) \sum_{\mathbf{G}>0} e^{-\frac{1}{2} \sigma_{i}^{2} G^{2}} \frac{e^{i \mathbf{G}\left(\mathbf{x}-\mathbf{x}_{i}\right)}}{|\mathbf{G}|^{2}}
$$

carrying a sum over the ions indexed with $i$ at positions $\mathbf{x}_{i}$.

The use of this Gaussian approximation requires a little discussion. First, it should be noted that the use of the sum over only the discrete wave vectors $\mathbf{G}$ instead of an integral is perfectly legitimate: it corresponds to the fact that we are not representing only a single ion with each term but rather an infinite periodic array of them arranged in a crystal lattice. This means that all terms of $\rho_{I}$ that do not lie on points of the reciprocal lattice go to zero. We can also note that obviously the resulting charge distribution does not likely resemble the real one. However, this charge distribution is positive, as $Z_{i}>N_{i}$ for all ions, and thus repulsive to the muons. Since we are interested only in the minima of the potential for the muon, we can be sure that they will be as far as possible from these lumps of positive charge. In fact, the details of the shape would matter much more for the potential at short range-from afar, these will act mostly like point charges as their size is still rather small compared to the overall volume of the cell.

Finally, we consider the problem of the cutoff over reciprocal space vectors. While for $\rho_{e}$, this is exact as the finite grid is the one that CASTEP itself used to compute it in the first place, for $\rho_{I}$, it is effectively a truncation of an infinite series. The crucial parameter becomes then $\sigma_{i}$. If this is big enough, then the Gaussian term in Eq. (7) falls off before the cutoff is even reached. If it is smaller, then there is a risk of the truncation having a sensible effect on the charge density and thus on the potential, producing spurious undulatory behavior. At the moment, it is up to the user to diagnose and counteract these problems. The parameter $s$ that controls the scale of $\sigma_{i}$ can be adjusted to fix any issues depending on the specifics of the system of interest; in general, we found that a default value of $s=5$ tends to work well in most cases.

\section{SOFTWARE IMPLEMENTATION}

Both the symmetry analysis and the UEP implementation described here can be found within a Python library we deployed specifically to aid muon computational science, pymuon-suite. The library can be found on Github ${ }^{13}$ and is released under a GNU v3.0 open source license. The library depends on a few other libraries to work properly. The most important ones are Numpy, ${ }^{14}$ Scipy, ${ }^{15}$ the Atomic Simulation Environment, ${ }^{16}$ Spglib, ${ }^{17}$ and Soprano. ${ }^{18}$ All of these libraries are available on the Python Package Index and thus can be installed automatically together with pymuon-suite without any additional effort on the user's part.

Once installed, pymuon-suite provides the user both with a Python API to use for custom programs and with a series of prepackaged scripts that perform the most common operations. Four of these scripts are relevant to the tools described in this work:

- pm-symmetry performs the analysis of special Wyckoff positions described in Sec. II A;

- pm-uep-opt performs an optimization of the muon position under the unperturbed electrostatic potential;

- pm-uep-plot produces 1D and 2D ASCII files describing the UEP along paths or on specified planes useful to produce plots; and

- pm-muairss produces batches of structure files with a muon defect added following a Poisson random distribution and analyses and clusters the results of their optimization.

The symmetry script is particularly simple as it does not need any parameters, and it can be run simply by executing the command

pm-symmetry <structure file>

in which the structure file has to be any supported crystallographic file format (such as .cif or .cell). For the UEP scripts, instead, 
TABLE I. Experimental and theoretical determination of the $\mu^{+}$stopping site in the samples studied in this work. The cluster column describes the fraction of muonated structures in the clusters representing the muon stopping site. The predicted site that agrees with the experimental result is the one with the largest fraction of structures.

\begin{tabular}{|c|c|c|c|}
\hline Sample & Experimental sites & Theoretical sites (UEP) & Clusters \\
\hline $\mathrm{Cu} \mathrm{fcc}$ & Octahedral $^{\mathrm{a}}$ & Octahedral $(\mathrm{O})$ and tetrahedral $(\mathrm{T})$ & $\mathrm{O}\left(\frac{67}{103}\right), \mathrm{T}\left(\frac{36}{103}\right)$ \\
\hline $\mathrm{TiO}_{2}$ rutile & Close to $\mathrm{O}_{\text {apical }}$ and $\mathrm{O}_{\text {planar }}{ }^{\mathrm{b}}$ & Close to $\mathrm{O}_{\text {apical }}$ & $\mathrm{O}_{\text {apical }}\left(\frac{559}{563}\right)$ \\
\hline $\mathrm{MnSi}$ & 4a-I Wyckoff & $\mathrm{S}, \mathrm{A}_{1}, \mathrm{~A}_{2}, \mathrm{~A}_{3}$ & $S\left(\frac{230}{423}\right), A_{1}\left(\frac{104}{423}\right), A_{2}\left(\frac{67}{423}\right), A_{3}\left(\frac{22}{423}\right)$ \\
\hline $\mathrm{Fe}_{3} \mathrm{O}_{4}$ magnetite & Close to $\mathrm{O}$ in planes $\perp$ to $\langle 111\rangle^{\mathrm{d}}$ & $\mathrm{O}_{\langle 111\rangle}$ and cubic $(\mathrm{C})$ & $\mathrm{O}_{\langle 111\rangle}\left(\frac{139}{152}\right), \mathrm{C}\left(\frac{13}{152}\right)$ \\
\hline $\mathrm{LiF}$ & $\mathrm{F} \mu \mathrm{F}$ state $\mathrm{e}^{\mathrm{e}}$ & Tetrahedral site $(\mathrm{T})$ and vertex site $(\mathrm{V})$ & $\mathrm{T}\left(\frac{1}{42}\right), \mathrm{V}\left(\frac{41}{42}\right)$ \\
\hline
\end{tabular}

${ }^{\mathrm{a}}$ Level-crossing measurements, M15 muon channel at TRIUMF (Canada). T $=40 \mathrm{~K}$ and $156 \mathrm{~K}, \mathrm{~B}=0.012 \mathrm{~T}$.

${ }^{\mathrm{b}}$ Transverse field $\mu S R$, MUSR instrument at ISIS (UK). T $=[1.2 \mathrm{~K}-10 \mathrm{~K}], \mathrm{B}=0.02 \mathrm{~T}^{22}$

${ }^{c}$ Transverse field $\mu S R$, GPS instrument at PSI (Switzerland). $\mathrm{T}=50 \mathrm{~K}, \mathrm{~B}=0.52 \mathrm{~T} .{ }^{2}$

${ }^{\mathrm{d}}$ Transverse field $\mu S R$, muon channel at LAMPF (US). T $=298 \mathrm{~K}, \mathrm{~B}=0.4 \mathrm{~T}$. ${ }^{24}$

${ }^{\mathrm{e}}$ Zero field $\mu S R, \mathrm{M} 15$ muon channel at TRIUMF (Canada). $\mathrm{T}=80 \mathrm{~K} .{ }^{25}$

input files in the YAML format ${ }^{19}$ containing parameters are necessary. These scripts run with

$$
\begin{aligned}
& \text { pm-uep-plot <parameters file> } \\
& \text { pm-uep-opt <parameters file> }
\end{aligned}
$$

Finally, pm-muairss operates similarly but has both a "write" and a "read" mode, one to create structure files and the other to interpret the results of their optimization. It also needs both a structure and a parameter file as inputs. To avoid mistakenly overwriting important data, the default mode is read. The two modes are used as follows:

pm-muairss -t w <structure file $><$ parameters file $>$

\# To write

pm-muairss -t $r<$ structure file $><$ parameters file $>$ \# To read

The -t $r$ argument is optional; if omitted, read mode is assumed. For parameter files, each script has its own arguments that can be set using them. The specific accepted parameters and their meaning for each script are detailed in Appendix C.

An example of a possible work pipeline to use the UEP method could then be as follows:

- run a CASTEP calculation on a bulk structure (non muonated) to compute the electronic density to use as input for the UEP method;

- generate a number of muonated structures with a random distribution of starting positions with pm-muairss in the write mode;
- relax each of these structures with pm-uep-opt; and

- perform a cluster analysis on the relaxed structures to identify the muon stopping sites with pm-muairss in the read mode.

The clusters with the largest number of muons will then roughly correspond to the minima of the electrostatic potential with the largest attraction basin.

\section{EXAMPLE SYSTEMS}

Table I compiles the muon stopping sites predicted by experiments and the UEP method for all the materials studied in this work. We chose examples of different nature: copper, which is a metal; rutile $\mathrm{TiO}_{2}$, a semiconductor; $\mathrm{MnSi}$ manganese silicide, a material that exhibits a homochiral spin spiral structure; $\mathrm{Fe}_{3} \mathrm{O}_{4}$ magnetite, a ferrimagnetic oxide; and $\mathrm{LiF}$, an insulator. In all of these materials, the muon stopping site has been unequivocally determined by experimental methods. Table II shows the main UEP parameters used for determining the muon stopping site for all the examples simulated in this work.

In copper, the muon stopping site was experimentally determined to be at the center of a copper octahedron in the copper fcc crystalline lattice. Level-crossing measurements were performed at the TRIUMF muon source (Canada) for samples at temperatures of $40 \mathrm{~K}$ and $156 \mathrm{~K}$ and longitudinal magnetic fields ranging from $0 \mathrm{~T}$ to $0.012 \mathrm{~T}^{20,21}$ The UEP method predicted two stopping sites

\begin{tabular}{|c|c|c|c|c|}
\hline Sample & Poisson $(\AA)$ & vdw_scale & uep_gw_factor & clustering_hier_t \\
\hline $\mathrm{Cu} \mathrm{fcc}$ & 0.4 & 0.25 & 4.0 & 0.2 \\
\hline $\mathrm{TiO}_{2}$ rutile & 0.4 & 0.25 & 5.0 & 0.2 \\
\hline $\mathrm{MnSi}$ & 0.5 & 0.3 & 5.0 & 0.2 \\
\hline $\mathrm{Fe}_{3} \mathrm{O}_{4}$ mag. & 0.8 & 0.5 & 6.0 & 0.2 \\
\hline $\mathrm{LiF}$ & 0.6 & 0.25 & 4.0 & 0.2 \\
\hline
\end{tabular}

TABLE II. Unperturbed electrostatic potential technical details for all the samples simulated in this work 
(T)

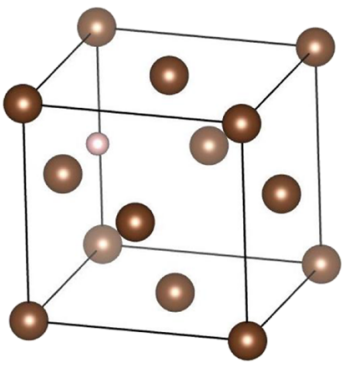

(O)

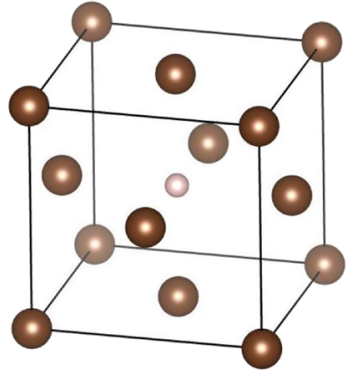

FIG. 1. Predicted (a) tetragonal (T) and (b) octahedral (O) $\mu$ stopping sites in $\mathrm{Cu}$ bcc.

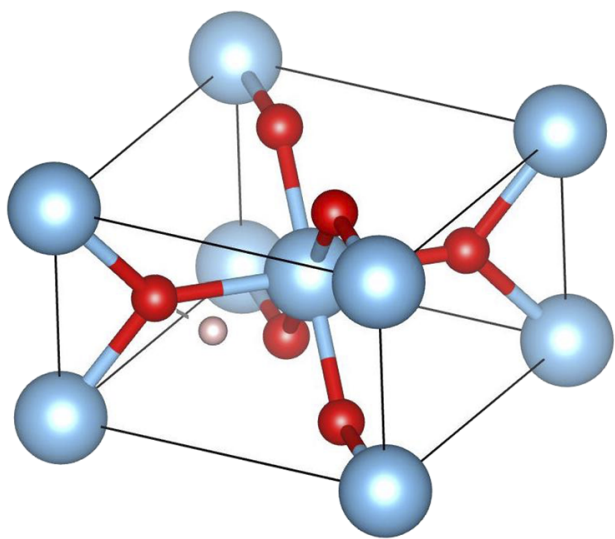

FIG. 2. Predicted $\mu$ stopping site in $\mathrm{TiO}_{2}$ rutile. $\mu$ close to $\mathrm{O}_{\text {apical }}$ with $\mathrm{OH}$ line in the ab plane.

in fcc copper: one in a tetrahedral site and the other in an octahedral site (Fig. 1). The cluster with the largest number of structures and the lowest average energy is the one that places the muon in the octahedral site.

In $\mathrm{TiO}_{2}$ rutile, transverse field $\mu S R$ measurements performed in the MUSR instrument at ISIS(UK) ${ }^{22}$ identified muon stopping sites where the muon has a low temperature ground state and a high temperature excited state, both corresponding to a muon bound to one of the six $\mathrm{O}$ atoms that form an octahedron around the $\mathrm{Ti}^{3+}$
(C)

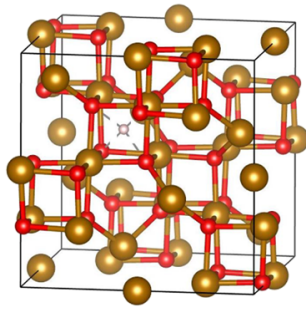

$\mathrm{O}_{[111]}$

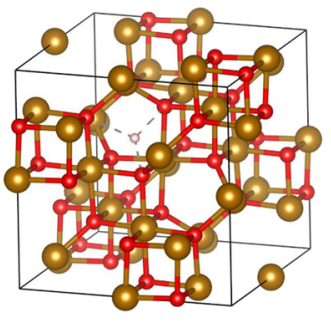

FIG. 4. Predicted $\mathrm{O}_{[111]}$ and cubic (C) $\mu$ stopping sites in $\mathrm{Fe}_{3} \mathrm{O}_{4}$.
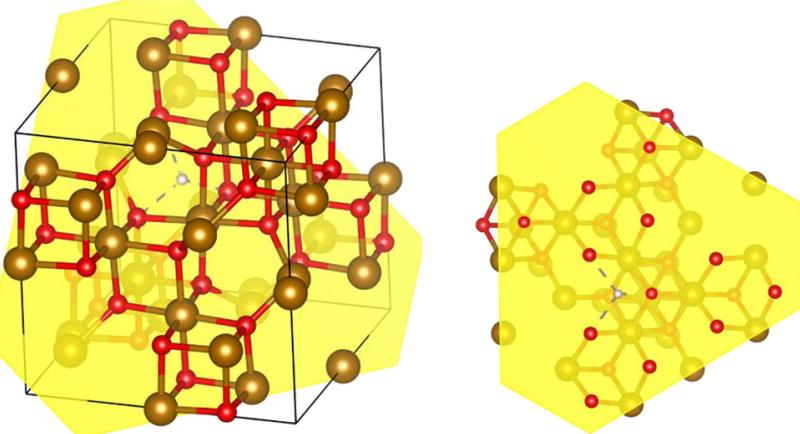

FIG. 5. Planar region for the predicted $\mathrm{O}_{\langle 111\rangle} \mu$ stopping site in $\mathrm{Fe}_{3} \mathrm{O}_{4}$.

at the center of the $\mathrm{TiO}_{2}$ rutile unit cell. Each one of these stopping sites has a different $\mathrm{O}-\mathrm{Ti}^{3+}$ bonding configuration, with the ground state formed by bonding the muon to the in-plane oxygen atoms that lie in the same plane as $\mathrm{Ti}^{3+}$. These two sites are related by symmetry and are only distinguished by the electronic structure of the $\mathrm{TiO}_{2}$ rutile. The cluster with the largest number of structures and the lowest average energy predicted by the UEP method is the one that places the muon at the ground state described above (Fig. 2).

Regarding $\mathrm{MnSi}$, transverse field $\mu \mathrm{SR}$ experiments carried out at the GPS instrument in PSI (Switzerland) ${ }^{23}$ identified the stopping site of the muon to be along the 4a-I Wyckoff axis of symmetry, in the MnSi unit cell. The stopping site was identified to have the fractional coordinates given by $(0.532,0.532,0.532)$. The UEP method predicted four potential stopping sites: a highly symmetric one (S)
(S)

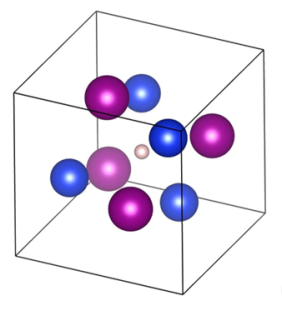

$\left(\mathrm{A}_{1}\right)$

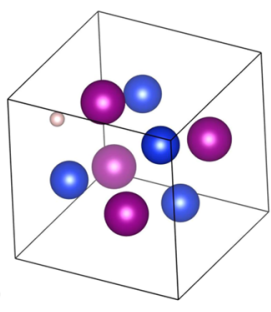

$\left(\mathrm{A}_{2}\right)$

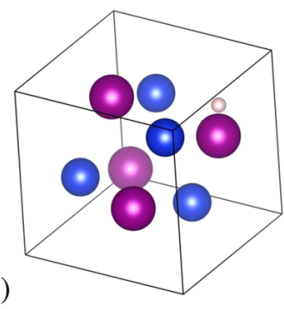

$\left(\mathrm{A}_{3}\right)$

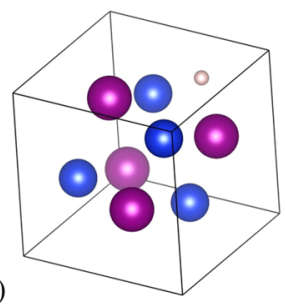

FIG. 3. Predicted $S, A_{1}, A_{2}$, and $A_{3} \mu$ stopping sites in $\mathrm{MnSi}$. 
(T)

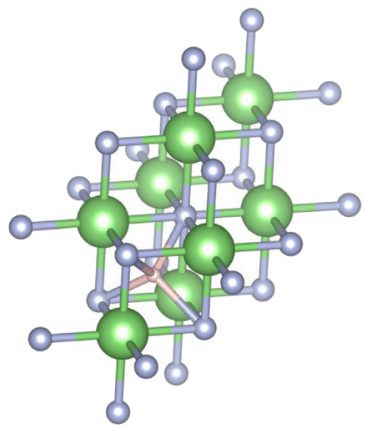

(V)

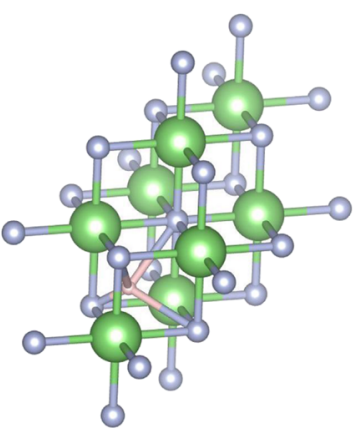

FIG. 6. Predicted (T) tetragonal and vertex (V) $\mu$ stopping sites in LiF.

and three others, namely, $A_{1}, A_{2}$, and $A_{3}$ (Fig. 3). The $S$ site, which originates from the cluster with the largest number of structures predicted by the UEP, agrees with the experimentally observed site.

Potential muon stopping sites in $\mathrm{Fe}_{3} \mathrm{O}_{4}$ magnetite were found using transverse field $\mu S R$ experiments performed at LAMPF (US). ${ }^{2}$
The stopping sites are (a) located within a planar region that is perpendicular to the $\langle 111\rangle$ direction and (b) situated within $\approx 1.5 \AA$ of one of the oxygen atoms defining the planar region. Figures (4) and (5) show the $\mathrm{O}_{\langle 111\rangle}$ site predicted by the UEP method. An example of a planar region perpendicular to the $\langle 111\rangle$ direction is indicated in yellow. The muon is located at $\approx 1.3 \AA$ from its closest oxygen atom in the planar region.

Finally, the behavior of $\mu$ in LiF has been studied using zero field $\mu S R$ experiments performed at the M15 muon channel at TRIUMF in Canada. ${ }^{25}$ The stopping site is located between two $\mathrm{F}$ atoms forming the distinctive $\mathrm{F} \mu \mathrm{F}$ center. None of the predicted stopping sites are, however, in the $\mathrm{F} \mu \mathrm{F}$ center: they are contained within a tetrahedron defined by four $\mathrm{F}$ atoms and are shown in Fig. (6). Site (T) is at the center of the tetrahedron, while site (V) is displaced from the tetrahedron's center and closer to one of its vertices. $\mathrm{LiF}$ is a case example of the strong influence that the muon can have on the atomic positions of the host atoms in its vicinity. In the $\mathrm{F} \mu \mathrm{F}$ center, the $\mathrm{F}-\mu$ bonds shorten the length between the $\mathrm{F}$ atoms by $\sim 20 \%$. The UEP method cannot account for that, and consequently, the predicted stopping sites are not the experimental ones.

TABLE III. Symmetry analysis results for all the samples simulated in this work.

\begin{tabular}{lcc}
\hline \hline Sample & UEP & Wyckoff positions \\
\hline $\mathrm{Cu} \mathrm{fcc}$ & $\mathrm{O}$ and $\mathrm{T}$ & 40 positions including O and $\mathrm{T}$ \\
$\mathrm{TiO}_{2}$ rutile & $\mathrm{O}_{\text {apical }}$ and $\mathrm{O}_{\text {planar }}$ & 10 positions including $\mathrm{O}_{\text {apical }}$ and $\mathrm{O}_{\text {planar }}$ \\
$\mathrm{MnSi}$ & $\mathrm{S}, A_{1}, A_{2}, A_{3}$ & No empty Wyckoff position \\
$\mathrm{Fe}_{3} \mathrm{O}_{4}$ mag. & $\mathrm{O}_{\langle 111\rangle}$ and $\mathrm{C}$ & 24 positions including C \\
$\mathrm{LiF}$ & $\mathrm{T}$ and $\mathrm{V}$ & 8 positions including $\mathrm{T}$ \\
\hline
\end{tabular}

${ }^{a}$ The pm-symmetry software only identifies Wyckoff positions with zero degrees of freedom, i.e., points. MnSi has no Wyckoff points: it has the 4a-I Wyckoff axis.

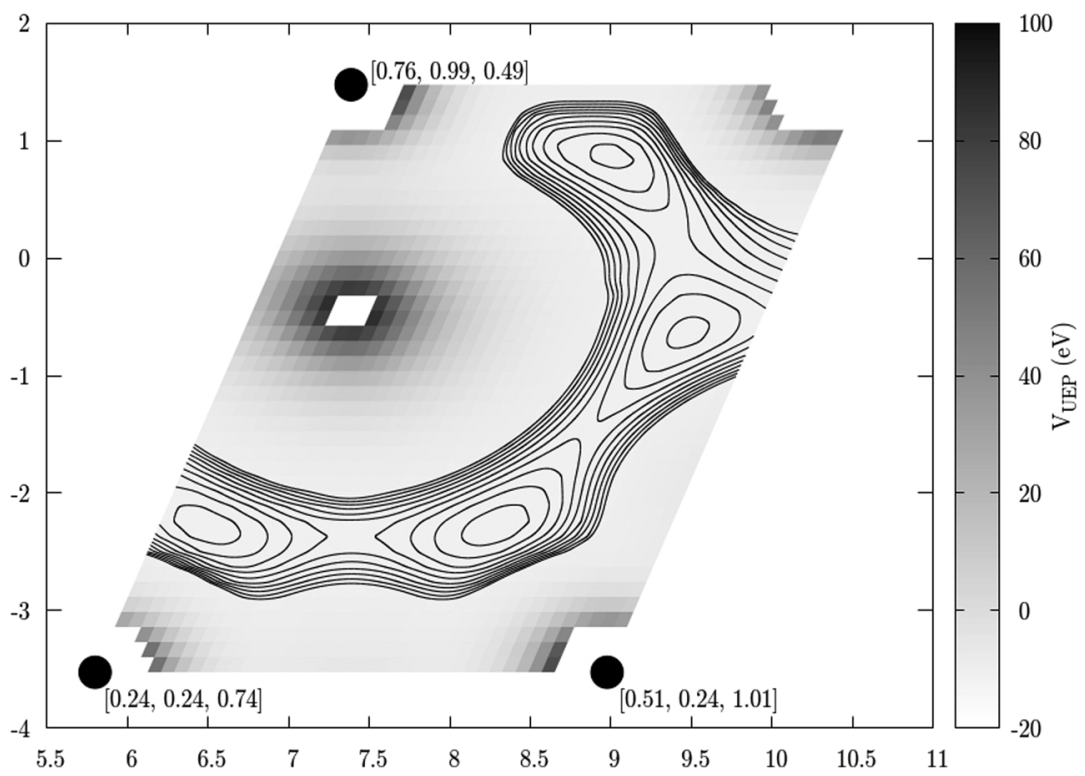

FIG. 7. UEP in the planar region perpendicular to $\langle 111\rangle$ in $\mathrm{Fe}_{3} \mathrm{O}_{4}$. The black dots indicate the three closest oxygen atoms to the predicted muon stopping site. 
For all these example systems, we also performed a symmetry analysis of all their Wyckoff positions using the pm-symmetry library, which are shown in Table III.

As we can see, many of the muon stopping sites theoretically predicted by the UEP method can be hinted at by performing a very simple calculation using the pm-symmetry library. Similarly, we can run the pm-uep-plot library and obtain graphic files that describe the UEP in a specified region of the unit cell. An example of these UEP plots is shown in Fig. (7), which corresponds to the planar region in $\mathrm{Fe}_{3} \mathrm{O}_{4}$ that is perpendicular to the $\langle 111\rangle$ direction and and is indicated in yellow in Fig. (5).

\section{CONCLUSIONS}

In this work, we have presented, described, and tested our new Python software package pymuon-suite and its various associated utilities, which can be used to study the potential muon stopping sites in crystalline materials. We have also shown that there is a connection between the symmetry properties of the crystalline material's electrostatic potential and the potential muon stopping sites in that material. Finally, the examples studied in this work show that our version of the UEP method can reliably predict the muon stopping sites in a variety of materials using purely theoretical means and reasonably cheap computer simulations.

\section{SUPPLEMENTARY MATERIAL}

In the supplementary material, there are examples of input and output files for the pm-symmetry, pm-muairss, and pm-uep-opt libraries that correspond to the search of the muon stopping sites in $\mathrm{Fe}_{3} \mathrm{O}_{4}$. These examples are organized so as to explain, with a practical example, how the search of the muon stopping sites could be carried out.

\section{ACKNOWLEDGMENTS}

The authors are grateful for the computational support provided by (a) the STFC Scientific Computing Department's SCARF cluster and (b) the UK Materials and Molecular Modelling Hub for computational resources, which is partially funded by EPSRC (Grant No. EP/P020194/1). Funding for this work was provided by the STFC-ISIS muon source, by the Ada Lovelace Centre at SCD, and by the CCP for NMR Crystallography, funded by EPSRC under Grant Nos. EP/J010510/1 and EP/M022501/1.

\section{APPENDIX A: PROPERTIES OF THE HESSIAN AT SPECIAL WYCKOFF POSITIONS}

Let us consider the Hessian $H$ of a generic function with space group symmetry $\mathscr{G}$ at a special Wyckoff position $X$, invariant under all symmetry operations $g \in S_{X}$. From similar considerations as in Eq. (2), we can find that, in general, the Hessian transforms as

$$
H=\mathbf{W}^{T} H^{\prime} \mathbf{W} .
$$

Again, at $X$, it must be that $H=H^{\prime}$ for all the operations under which $X$ is fixed. We know that the Hessian must be a symmetric matrix. We can then split it in an isotropic part and a traceless symmetric tensor,

$$
\begin{aligned}
H & =h_{\text {iso }} \mathbf{W}^{T} \mathbf{W}+\mathbf{W}^{T} H^{(\text {symm })} \mathbf{W} \\
& =h_{\text {iso }} \mathbb{I}+\mathbf{W}^{T} H^{(\text {symm })} \mathbf{W} \quad \forall(\mathbf{W}, \mathbf{w}) \in S_{X},
\end{aligned}
$$

with $h_{\text {iso }}=\operatorname{Tr}(H) / 3$ and where we made use of the fact that if $X$ is a special Wyckoff position, $S_{X}$ can only contain reflections, rotations, inversions, and rotoinversions, all of which have orthogonal transformation matrices (in general, this is not necessarily as given). This leaves us with the following relation:

$$
H^{(\text {symm })}=\mathbf{W}^{T} H^{(\text {symm })} \mathbf{W} \quad \forall(\mathbf{W}, \mathbf{w}) \in S_{X} .
$$

We can recast this problem in a way that is very similar to Eq. (3). This is fundamentally a system of nine equations in nine variables,

$$
H_{i j}^{(\text {symm })}=W_{i l} W_{j k} H_{k l}^{(s y m m)} .
$$

However, since we know that $H^{(\text {symm })}$ is both symmetric and traceless, it only has five independent components we care about, and four of the equations are just linear combinations of the other. We can then write this as a system of five equations in five variables in the matrix form,

$$
\left(\mathbf{W}^{(5)}-\mathbb{I}\right) H^{(5)}=0 \quad \forall(\mathbf{W}, \mathbf{w}) \in S_{X},
$$

where $H^{(5)}$ is a column vector containing the five independent components in any order we like, and the precise form of $\mathbf{W}^{(5)}$ depends on the convention we chose. One can then check for each special Wyckoff position whether there is any vector that satisfies all these conditions. The principle is the same as in Eq. (3) - we seek whether there is at least one axis that is invariant under all $\mathbf{W}^{(5)}$. If there is none, then $H^{(5)}$ must be necessarily zero, which means that the Hessian will be isotropic at $\mathrm{X}, H(X)=h_{\text {iso }}(X) \mathbb{I}$.

One here must pay attention. All of these considerations apply to $X$ expressed in fractional coordinates, the ones for which the operations in $\mathscr{G}$ are written. The Hessian, too, includes the derivatives of the function with respect to the fractional coordinates. In the case of a space group with a cubic lattice, all considerations immediately apply to the Hessian in cartesian coordinates too, and one only has to divide $h_{\text {iso }}$ by the square of the lattice parameter $a$. However, in general, if we have a set of lattice vectors $\mathbf{C}$ such that the Cartesian coordinates of the Wyckoff position are $X_{C}=\mathbf{C X}$, then the isotropic Hessian transforms as

$$
H_{C}=\left(\mathbf{C}^{-1}\right)^{T} H \mathbf{C}^{-1}=h_{\text {iso }}\left(\mathbf{C}^{-1}\right)^{T} \mathbf{C}^{-1} .
$$

It then depends on the properties of the matrix $\left(\mathbf{C}^{-1}\right)^{T} \mathbf{C}^{-1}$ what the Hessian is like in Cartesian coordinates. If the cell is cubic, then it will remain isotropic, as said above. If it is orthorhombic, then it will not be isotropic but still be positive or negative definite. In general, if $\left(\mathbf{C}^{-1}\right)^{T} \mathbf{C}^{-1}$ is definite, then so will be the Hessian. Conversely, if it is not, we can then guarantee that the Hessian will not be either, which would make the special Wyckoff position a saddle point of the function.

\section{APPENDIX B: TECHNICAL DETAILS OF CALCULATIONS}

The DFT-based computer simulations carried out in this work were performed with the $\mathrm{CASTEP}^{26}$ code. The plane wave cutoff, 
TABLE IV. CASTEP technical details for all the samples simulated in this work.

\begin{tabular}{lccc}
\hline \hline Sample & $\mathrm{E}_{\text {cutoff }}(\mathrm{eV})$ & k-point grid & XC funct. \\
\hline $\mathrm{Cu} \mathrm{fcc}$ & 500.0 & $4 \times 4 \times 4$ & $\mathrm{PBE}$ \\
$\mathrm{TiO}_{2}$ rutile & 700.0 & $2 \times 2 \times 2$ & $\mathrm{PBE}$ \\
$\mathrm{MnSi}_{\mathrm{Fe}_{3} \mathrm{O}_{4} \text { mag. }}^{700.0}$ & $3 \times 3 \times 3$ & $\mathrm{PBE}$ \\
$\mathrm{LiF}$ & 800.0 & $3 \times 3 \times 3$ & $\mathrm{PBE}$ \\
& 700.0 & $3 \times 3 \times 3$ & PBE \\
\hline
\end{tabular}

$\mathrm{E}_{\text {cutoff, }}$ for these calculations was chosen by converging energy and forces. This was done using the automated tool CASTEPconv ${ }^{6}$ to try a range of possible values, with every other condition fixed. The final choices were the values for which any successive refinement yielded a difference in energy and forces lower than a fixed tolerance. This was taken to be smaller than the tolerances used for self-consistent field and geometry optimization calculations. As regards the k-point grid size, Monkhorst-Pack ${ }^{27} \mathrm{k}$-point grids were used. This produced forces accurate well within an error of $0.05 \mathrm{eV} / \AA$, which was used as the limit tolerance for geometry optimization.

Geometry optimization on these structures were performed with a LBFGS algorithm, with fixed unit cell parameters, to a tolerance of $0.05 \mathrm{eV} / \AA$ for the forces. The PBE exchange-correlation functional was used in combination with auto-generated ultrasoft pseudopotentials.

Table IV shows the main parameters used in the CASTEP calculations performed in this work.

\section{APPENDIX C: PARAMETERS USED . yaml INPUT FILES}

Here, we list the keywords for the input files of the pymuonsuite scripts used in this work. The scripts are the ones listed in Sec. III. The keywords are then used in a file written in the YAML format, namely, a plain text file with rows written in the format $<$ keyword $>$ : $<$ value $>$

\section{Unperturbed electrostatic potential optimization: pm-uep-opt}

Here are listed all the keywords used to run the pm-uep-opt script, used for optimization of the muon position under the unperturbed electrostatic potential approximation.

- chden_path: path of the folder in which the charge density file produced by a CASTEP calculation (extension.den_fmt) to use for the UEP can be found. Default:. Type: string;

- chden_seed: seedname of the charge density file produced by a CASTEP calculation (extension . den_fmt) to use for the UEP. In combination with the previous keyword, the file will be searched as $\langle$ chden_path $\rangle /\langle$ chden_seed $>$.den_fmt. Default: NONE Type: string;

- gw_factor: Gaussian width factor used to define the size of the ionic charges by scaling the pseudopotential radius. Corresponds to the $s$ factor, as described in Sec. II B. Default: 5.0 TYPE: float;
- mu_pos: starting position of the muon in the unit cell, expressed in absolute coordinates, in $\AA$. Default: [0.0, $0.0,0.0]$ TyPE: [float];

- geom_steps: maximum number of geometry optimization steps. Default: 30 Type: int;

- opt_tol: force tolerance for each geometry optimization in eV/Å. Default: 1E-5 Type: float;

- opt_method: method used for geometry optimization. Corresponds to one of the methods used by Scipy's scipy .optimize.minimize function. See the documentation for the options. Default: trust-exact Type: string;

- particle_mass: mass of the particle, in kg. Important for zero point energy estimation. By default, it is the mass of the muon. Default: 1.884E-28 Type: float; and

- save_pickle: If True, save the output result in a pickled file for further reading and reusing with other Python scripts. Default: true Type: boolean.

\section{Unperturbed electrostatic potential plotting: pm-uep-plot}

Here are listed all the keywords used to run the pm-uep-plot script, used for plotting the unperturbed electrostatic potential along directions and planes in the unit cell. We omit explaining the keywords chden_path, chden_seed, and gw_factor, which are in common with pm-uep-opt and work as explained in Subsection 1 of Appendix C.

- line_plots: specify one or more line segments along which to plot the value of the UEP. Each line segment is specified by a list, and there are a number of possible methods to specify them:

- crystallographic direction, starting point, length, and number of points, for example,

$$
-[[1,1,0],[0,0,0], 10,100]
$$

will produce a plot along the [110] direction, starting from the origin, continuing for $10 \AA$ and with 100 points spaced $0.1 \AA$ each;

- starting point, end point, and number of points, for example, $-[[0,0,0],[1,1,1], 100]$

will produce a plot sampling the vector connecting the origin with the position $[1,1,1] \AA$ in the cell (these are absolute positions), with a grid of 100 points;

- starting atom, end atom, and number of points; for example, $-[1,2,20]$

will produce a plot sampling the line connecting the atoms with indices 1 and 2 in the structure file, split in 20 points.

- plane_plots: specify one or more planes along which to plot the value of the UEP. Each plane is specified by a list, and there are a number of possible methods to specify them:

- three corners, points along width, and points along height, for example,

$$
-[[0,0,0],[3,0,0],[0,0,3], 20,20]
$$


in a cubic lattice with $a=3$ would produce a plot of the $x z$ face of the cell, split into a $20 \times 20$ grid,

- three atom indices to act as corners, points along width, and points along height, for example,

- $[0,1,2,20,20]$

would produce a parallelogram having the vector connecting atoms 0 and 1 as base, the vector connecting atoms 0 and 2 as side, and 20 points along each side for a $20 \times 20$ overall grid.

\section{Random structure generation: pm-muairss}

Here are listed all the keywords used to run the pm-muairss script. For completeness, words that are relevant to usage with CASTEP or DFTB+ for structure optimization are included too, though they are not relevant for the current work.

- name: name to call the folder for containing each structure. This name will be postfixed with a unique number, e.g., struct_001. Default: struct Type: string;

- calculator: calculator(s) used to optimize the muon position. Must be a comma separated list of values. Currently supported calculators are CASTEP, DFTB+, and UEP. Can also pass ALL as an option to generate files for all calculators. Default: dftb+ Type: string;

- poisson_r: radius in $\AA$ for generating muon sites with the Poisson disk algorithm. This radius is the minimum distance at which two muons can be placed from each other when the muonated structures are generated. Default: 0.8 Type: float;

- uep_chden: CASTEP charge density file. seed.den_fmt. Default: NONE Type: string;

- uep_gw_factor: Gaussian width factor used to define the size of the ionic charges by scaling the pseudopotential radius. Corresponds to the $s$ factor, as described in Sec. II B. Default: 5. 0 Type: float;

- vdw_scale: scale factor to multiply the standard van der Waals radius of each atom in the system, used to determine the minimum distance allowed between a muon and other atoms. Bigger values will evacuate a larger sphere around the existing atoms. Default: 0.5 Type: float;

- charged: determines whether the implanted muons will be charged or neutral. Must be True to use UEP. Default: false TYPE: boolean;

- supercell: supercell size and shape to use. This can either be a single int, a list of three integers, or a $3 \times 3$ matrix of integers. For a single number, a diagonal matrix will be generated with the integer repeated on the diagonals. For a list of three numbers, a diagonal matrix will be generated where the diagonal elements are set to the list. A matrix will be used directly as is. Default is a $3 \times 3$ identity matrix. Default: identity TYPE: matrix;

- out_folder: name for the output folder used to store the structural input files generated. Default. /muon-airss-out TYPE: string;

- geom_steps: maximum number of geometry optimization steps. Default: 30 Type: int;

- geom_force_tol: force tolerance for each geometry optimization in $\overline{\mathrm{V}} / \mathrm{A}$. Default: 0.05 Type: float;
- clustering_method: clustering method to use to process results. The options are HIER (for hierarchical clustering) and KMEANS (for k-means clustering). Default: hier Type: string;

- clustering_hier_t: normalized $t$ parameter for hierarchical clustering. Higher $t$ will produce a smaller number of bigger clusters. Default: 0.3 Type: float;

- clustering_kmeans_k: expected number of clusters for kmeans clustering. Default: 4 Type: int;

- clustering_save_min: If True, save the minimum energy structure for each cluster as a separate file. Default: false TYPE: boolean;

- clustering_save_format: extension of file format in which to save the minimum energy structures if clustering _save_min is True. Default: cif Type: string;

- castep_command: command used to run the CASTEP executable on the system. Default: castep.serial Type: string;

- dftb_command: command used to run the DFTB+ executable on the system. Default: dftb+ Type: string;

- script_file: path of a submission script template to copy into each individual generated structure's folder for use with submission systems on HPC machines. Any literal instance of the string \{seedname\} will be replaced with the name of the structure in that folder, which allows us to create submission scripts for batches of CASTEP structures. Default: NONE TYPE: string;

- castep_param: path to a CASTEP parameter file to use for all calculations. Default: NONE TYPE: string;

- dftb_set: Slater-Koster parameterization to use with DFTB+. It determines which elements can be treated; see dftb.org for more details. Can currently be $30 \mathrm{ob}-3-1$ or pbc-0-3.Default: 3ob-3-1 Type: string;

- dftb_optionals: additional optional JSON files to activate for the DFTB+ parameterization. For example, including spinpol. json for 3ob-3-1 turns spin polarization on. Should be written like a list of strings (either in square brackets and comma separated or as a list using a as bullet and an entry on each line) Default: [] Type: [str];

- dftb_pbc: whether to turn on periodic boundary conditions in a DFTB+ calculation. Default: true Type: boolean;

- k_points_grid: k-points grid for periodic system calculations (CASTEP or DFTB+). Default: $[1,1,1]$ Type: [int]; and

- max_scc_steps: maximum number of self-consistent steps when converging the electronic wavefunction in either CASTEP or DFTB+. Default: 200 Type: int;

\section{DATA AVAILABILITY}

The data that support the findings of this study are available within the article and its supplementary material.

\section{REFERENCES}

${ }^{1}$ F. Bernardini, P. Bonfà, S. Massidda, and R. De Renzi, “Ab initio strategy for muon site assignment in wide band gap fluorides,” Phys. Rev. B 87, 115148 (2013).

${ }^{2}$ D. Cammarere, R. Scheicher, N. Sahoo, T. Das, and K. Nagamine, "First-principle determination of muon and muonium trapping sites in horse heart cytochrome $c$ 
and investigation of magnetic hyperfine properties," Physica B 289-290, 636-639 (2000).

${ }^{3}$ E. L. Silva, A. G. Marinopoulos, R. C. Vilão, R. B. L. Vieira, H. V. Alberto, J. Piroto Duarte, and J. M. Gil, "Hydrogen impurity in yttria: $A b$ initio and $\mu \mathrm{SR}$ perspectives," Phys. Rev. B 85, 165211 (2012).

${ }^{4}$ H. Maeter, H. Luetkens, Y. G. Pashkevich, A. Kwadrin, R. Khasanov, A. Amato, A. A. Gusev, K. V. Lamonova, D. A. Chervinskii, R. Klingeler, C. Hess, G. Behr, B. Büchner, and H.-H. Klauss, "Interplay of rare earth and iron magnetism in $\mathrm{RFeAsO}(\mathrm{R}=\mathrm{La}, \mathrm{Ce}, \mathrm{Pr}$, and $\mathrm{Sm})$ : Muon-spin relaxation study and symmetry analysis," Phys. Rev. B 80, 094524 (2009).

${ }^{5}$ L. Liborio, S. Sturniolo, and D. Jochym, "Computational prediction of muon stopping sites using ab initio random structure searching (AIRSS)," J. Chem. Phys. 148, 134114 (2018).

${ }^{6}$ S. Sturniolo, L. Liborio, and S. Jackson, "Comparison between density functional theory and density functional tight binding approaches for finding the muon stopping site in organic molecular crystals," J. Chem. Phys. 150, 154301 (2019).

${ }^{7}$ G. Seifert, D. Porezag, and T. Frauenheim, "Calculations of molecules, clusters, and solids with a simplified LCAO-DFT-LDA scheme," Int. J. Quantum Chem. 58, 185-192 (1996).

${ }^{8}$ M. Elstner, D. Porezag, G. Jungnickel, J. Elsner, M. Haugk, T. Frauenheim, S. Suhai, and G. Seifert, "Self-consistent-charge density-functional tight-binding method for simulations of complex materials properties," Phys. Rev. B 58, 72607268 (1998).

${ }^{9} \mathrm{P}$. Bonfa and R. De Renzi, "Toward the computational prediction of muon sites and interaction parameters," J. Phys. Soc. Jpn. 85, 091014 (2016).

${ }^{10}$ J. S. Möller, D. Ceresoli, T. Lancaster, N. Marzari, and S. J. Blundell, "Quantum states of muons in fluorides," Phys. Rev. B 87, 121108 (2013).

${ }^{11}$ B. Souvignier, H. Wondratschek, M. I. Aroyo, G. Chapuis, and A. M. Glazer, "Space groups and their descriptions," in International Tables for Crystallography (International Union of Crystallography, 2016), pp. 42-74.

${ }^{12}$ The Jacobian is a mathematical term that needs to be included in a three dimensional integral when the variables of the function being integrated are changed. In this case, the change of variables is from Cartesian coordinates to spherical coordinates.

${ }^{13}$ S. Sturniolo, L. Liborio, S. Jackson, and A. Laverack, pymuon-suite, https://github.com/muon-spectroscopy-computational-project/pymuon-suite, for the Github page of the Muon Spectroscopy Computational Project, where we deposited a collection of scripts and utilities for muon spectroscopy.

${ }^{14}$ S. van der Walt, S. C. Colbert, and G. Varoquaux, "The NumPy array: A structure for efficient numerical computation," Comput. Sci. Eng. 13, 22-30 (2011).

${ }^{15}$ T. E. Oliphant, "Python for scientific computing," Comput. Sci. Eng. 9, 10 (2007).
${ }^{16}$ A. H. Larsen, J. J. Mortensen, J. Blomqvist, I. E. Castelli, R. Christensen, M. Dułak, J. Friis, M. N. Groves, B. Hammer, C. Hargus, E. D. Hermes, P. C. Jennings, P. B. Jensen, J. Kermode, J. R. Kitchin, E. L. Kolsbjerg, J. Kubal, K. Kaasbjerg, S. Lysgaard, J. B. Maronsson, T. Maxson, T. Olsen, L. Pastewka, A. Peterson, C. Rostgaard, J. Schiøtz, O. Schütt, M. Strange, K. S. Thygesen, T. Vegge, L. Vilhelmsen, M. Walter, Z. Zeng, and K. W. Jacobsen, "The atomic simulation environment-A python library for working with atoms," J. Phys.: Condens. Matter 29, 273002 (2017).

${ }^{17}$ A. Togo and I. Tanaka, "Spglib: A software library for crystal symmetry search,” arXiv:1808.01590 (2018).

${ }^{18}$ S. Sturniolo, Soprano-A library to crack crystals, https://ccp-nc.github.io/ soprano/. The webpage corresponds to the github page of the Python library Soprano, which was developed by Simone Sturniolo. Soprano is a Python library developed and maintained by the CCP for NMR Crystallography as a tool to help scientists working with crystallography and simulations to generate, manipulate, run calculations on and analyze large data sets of crystal structures. It provides a number of functionalities to help automate many common tasks in computational crystallography.

${ }^{19}$ O. Ben-Kiki, C. Evans, and I. döt Net, YAML Ain't Markup Language $\left(\mathrm{YAML}^{\mathrm{TM}}\right.$ ) version 1.2, https://yaml.org/spec/1.2/spec.html, 2009.

${ }^{20}$ G. M. Luke, J. H. Brewer, S. R. Kreitzman, D. R. Noakes, M. Celio, R. Kadono, and E. J. Ansaldo, "Muon diffusion and spin dynamics in copper," Phys. Rev. B 43, 3284-3297 (1991)

${ }^{21}$ A. Schenck, "Static magnetic properties of metallic systems," in Muon Science: Muons in Physics, Chemistry and Materials (CRC Press, 1999).

${ }^{22}$ R. C. Vilão, R. B. L. Vieira, H. V. Alberto, J. M. Gil, A. Weidinger, R. L. Lichti, B. B. Baker, P. W. Mengyan, and J. S. Lord, "Muonium donor in rutile $\mathrm{Tio}_{2}$ and comparison with hydrogen," Phys. Rev. B 92, 081202 (2015).

${ }^{23}$ A. Amato, P. Dalmas de Réotier, D. Andreica, A. Yaouanc, A. Suter, G. Lapertot, I. M. Pop, E. Morenzoni, P. Bonfà, F. Bernardini, and R. De Renzi, "Understanding the $\mu \mathrm{SR}$ spectra of MnSi without magnetic polarons," Phys. Rev. B 89, 184425 (2014).

${ }^{24}$ C. Boekema, A. B. Denison, D. W. Cooke, R. H. Heffner, R. L. Hutson, M. Leon, and M. E. Schillaci, "Hyperfine field calculations: Search for muon stopping sites in $\mathrm{Fe}_{3} \mathrm{O}_{4}$," Hyperfine Interact. 15, 529 (1983).

${ }^{25}$ J. H. Brewer, S. R. Kreitzman, D. R. Noakes, E. J. Ansaldo, D. R. Harshman, and R. Keitel, "Observation of muon-fluorine "hydrogen bonding" in ionic crystals," Phys. Rev. B 33, 7813-7816 (1986).

${ }^{26}$ M. D. Segall, P. J. D. Lindan, M. J. Probert, C. J. Pickard, P. J. Hasnip, S. J. Clark, and M. C. Payne, "First-principles simulation:ideas, illustrations and the castep code," J. Phys.: Condens. Matter 14, 2717-2744 (2002).

${ }^{27}$ H. J. Monkhorst and J. D. Pack, "Special points for brillouin-zone integrations," Phys. Rev. B 13, 5188-5192 (1976). 